\title{
Local immune response in bladder pain syndrome/interstitial cystitis ESSIC type 3C
}

\author{
Marianne Gamper • Volker Viereck • Jakob Eberhard • \\ Jochen Binder • Carlo Moll • JoEllen Welter • \\ René Moser
}

Received: 4 January 2013 / Accepted: 6 April 2013 /Published online: 14 May 2013

(C) The Author(s) 2013. This article is published with open access at Springerlink.com

\begin{abstract}
Introduction and hypothesis Bladder pain syndrome/interstitial cystitis (BPS/IC) is identified based on subjective symptoms which lead to heterogeneous patient populations. Previous studies using gene expression arrays for BPS/IC with Hunner's lesions [European Society for the Study of Interstitial Cystitis (ESSIC) type 3C], a subtype of the condition discernible by cystoscopy, have revealed characteristic immune responses and urothelial abnormalities. This current study aimed to further characterize this subtype using a gene expression panel. We hypothesized that B-cell activation with high levels of urinary antibody concentration would be found.

Methods Cold-cup bladder biopsies, catheterized urine and blood were collected from $15 \mathrm{BPS} / \mathrm{IC}$ ESSIC type 3C patients, 11 non-inflammatory overactive bladder $(\mathrm{OAB})$ patients and eight healthy controls. Gene expression in biopsies was quantified by real-time quantitative
\end{abstract}

\footnotetext{
M. Gamper $(\bowtie)$

IKBT, Institut für Klinische Biomedizinische

Forschung Thurgau, Lauchefeld 31,

9548 Matzingen, Switzerland

e-mail: marianne.gamper@stgag.ch

V. Viereck $\cdot$ J. Eberhard $\cdot J$. Welter

Department of Gynaecology and Obstetrics,

Kantonsspital Frauenfeld, Frauenfeld, Switzerland

J. Binder

Department of Urology, Kantonsspital Frauenfeld,

Frauenfeld, Switzerland

C. Moll

Institute for Pathology, Spital Thurgau AG,

Münsterlingen, Switzerland

R. Moser

IBR Inc., Institute for Biopharmaceutical Research, Matzingen, Switzerland
}

polymerase chain reaction (RT-qPCR), immunohistochemistry was performed on bladder tissue and urinary immunoglobulins $G$ and A were quantified by enzyme-linked immunosorbent assay. Statistical analyses included the Kruskal-Wallis test for non-parametric data and post hoc tests identified differences between groups.

Results High expression of T- and B-cell markers (CTLA4, CD20,CD79A,IGH@), low expression of urothelial markers (KRT20, UPK1B, UPK3A), focal lymphoid aggregates in the submucosa and high immunoglobulin concentration in urine were found exclusively in BPS/IC ESSIC type $3 \mathrm{C}$ patients. Results for $\mathrm{OAB}$ were in intermediate ranges between the other two groups and $U P K 1 B$ even reached significantly lower expression when compared to healthy controls.

Conclusions BPS/IC ESSIC type 3C is characterized by a local adaptive immune response with elevated urinary antibody concentrations. Quantification of urinary immunoglobulin levels could be used for a non-invasive diagnosis of BPS/IC ESSIC type 3C.

Keywords Bladder pain syndrome/interstitial cystitis . Diagnostic markers · Immune response · Overactive bladder . Urothelium

\section{Introduction}

Twenty years ago, the chronic bladder disease "interstitial cystitis" (IC) was widely regarded as an inflammatory condition. Published IC research in the early 1990s was based primarily on biopsy and histological investigations [1, 2]. However, a debate began during this same time about the need for cystoscopy and biopsy in order to accurately diagnose IC [3]. Patients presenting with clinical symptoms such as pain and urinary frequency, in the absence of any known 
cause, were commonly diagnosed with "IC syndrome" [3]. Due to the subjectivity of this definition, a diverse group of patients were identified with IC ranging from those with neuropathic changes to those having a severe inflammation with macroscopically obvious degeneration of the urothelium. These broad inclusion criteria have made it difficult to establish diagnostic markers for the disease.

A consensus definition of "bladder pain syndrome/ interstitial cystitis (BPS/IC)" was proposed by the European Society for the Study of Interstitial Cystitis (ESSIC). The diagnosis is based on chronic pelvic pain, pressure or discomfort perceived to be related to the urinary symptom accompanied by at least one other urinary symptom such as persistent urge to void or frequency [4]. Confusable diseases [i.e. carcinoma, infection, overactive bladder $(\mathrm{OAB})$ ] need to be excluded. The severity of BPS/IC is classified by the rating of cystoscopic and histopathological evidence [4]. The maximum cystoscopic severity is the finding of Hunner's lesions, and the maximum histopathological severity is the finding of inflammatory infiltrates and/or detrusor mastocytosis and/or granulation tissue and/or intrafascicular fibrosis. These maximum findings are referred to as "ESSIC type 3C" [4].

In an effort to more precisely define suitable BPS/IC diagnostic markers, our research team conducted a pilot study in 2009 comparing gene expression analysis (Affymetrix GeneChip ${ }^{\circledR}$ Human Genome U133 Plus 2.0) of bladder biopsies from five patients with BPS/IC ESSIC type $3 \mathrm{C}$ and six healthy controls [5]. Approximately 1,000 genes from more than 50,000 probe sets were found to have a BPS/IC to healthy expression ratio greater than two. The corresponding gene expression patterns were characteristic for immune system diseases, and they predicted chronic inflammation with B- and T-lymphocyte infiltration. In addition, the array data suggest an abnormal differentiation of the urothelium which is in agreement with earlier results [6-8]. Two urothelium-specific genes, cytokeratin 20 (KRT20) and uroplakin 1B (UPK1B), were found to be expressed at very low levels [5].

While gene expression arrays can provide a large amount of information, the process is expensive, time-consuming and requires specialized equipment. We investigated if it was possible to characterize Hunner's positive BPS/IC by the quantification of seven representative urothelial, B- and Tcell markers. We hypothesized that a local B-cell activation would lead to high levels of antibody concentration in urine.

\section{Materials and methods}

\section{Description of patients}

This prospective cohort study assigned patients recruited at one urogynaecological centre in Switzerland from 2006 to
2011 to three comparison groups: BPS/IC ESSIC type 3C (group 1, $n=15$ ), $\mathrm{OAB}$ (group 2, $n=11$ ) and healthy controls (group 3,n=8). Each woman reviewed and completed relevant study documentation approved by the local ethics commission including an informed consent form (reference 9900.013).

In order to detect markers, homogeneous groups of patients were required. Consequently, patients with predominant pain symptoms were selected based on their diagnosis with classic ulcerative IC or IC with Hunner's lesions that conformed to the BPS/IC ESSIC type 3C criteria [4]. "Hunner's lesions" were identified according to ESSIC [4] as distinctive, circumscript reddened mucosal areas with small vessels radiating towards a central scar that, upon bladder distension, will rupture and bleed. As a control group, we chose patients who had primary urgency symptoms and a cystoscopically non-inflamed bladder wall ("non-inflammatory OAB"). These patients represent a subgroup of the $\mathrm{OAB}$ condition since there are also reports of an association between $\mathrm{OAB}$ and inflammation [9-11]. Healthy control patients were identified as women with no bladder symptoms and who were hospitalized for a hysterectomy shortly before enrolment in the study. Patients with urinary tract infections were excluded.

Biopsy removal and real-time quantitative polymerase chain reaction (RT-qPCR)

Two cold-cup biopsies were taken from each patient and, when present, from the transition zone lesion/non-lesion. Thus, bladder biopsies included various amounts of urothelium, submucosa and muscularis propria (detrusor). Histopathological testing of one of these tissue samples included mast cell quantification. Detrusor mastocytosis was defined according to ESSIC [4] as mast cell counts greater than 28 mast cells $/ \mathrm{mm}^{2}$. The other biopsied tissue was preserved in RNAlater (76104, QIAGEN, Valencia, CA, USA) and used for RNA isolation and quantification as described in the report of our pilot study [5]. Reverse transcription and RT-qPCR were done with materials and equipment from Applied Biosystems (Life Technologies, Zug, Switzerland) as follows: TaqMan ${ }^{\circledR}$ Reverse Transcription Reagents (N8080234) for reverse transcription, inventoried Human TaqMan ${ }^{\circledR}$ Gene Expression Assays (4331182) and TaqMan ${ }^{\circledR}$ Universal PCR Master Mix (4364340) for RT-qPCR on an Applied Biosystems 7500 Real-Time PCR System. The following TaqMan ${ }^{\circledR}$ Gene Expression Assays (4331182) were analysed: cytotoxic T-lymphocyteassociated protein 4 (CTLA4, Hs03044418_m1) as a Tcell marker; $C D 20$ (membrane-spanning 4-domains, subfamily A, member 1 MS4A1, Hs00544819_m1) and 
CD79A molecule, immunoglobulin-associated alpha (CD79A, Hs00233566 m1) as B-cell markers; immunoglobulin heavy locus (IGH@, Hs00378230_g1) as a marker for antibody heavy chain gene expression; and uroplakin 1B (UPK1B, Hs00199583_m1), uroplakin 3A (UPK3A, Hs00199590_m1) and cytokeratin 20 (KRT20, Hs00300643_m1) as urothelial markers. RT-qPCR reactions were done in duplicates in a $25 \mu \mathrm{l}$ volume. Standard cycling conditions were used $\left(2 \min 50{ }^{\circ} \mathrm{C}\right.$, $10 \min 95{ }^{\circ} \mathrm{C}, 40$ cycles $15 \mathrm{~s} 95{ }^{\circ} \mathrm{C} / 1 \min 60^{\circ} \mathrm{C}$ ). Relative gene expression (comparative $\mathrm{Ct}$ method quantitation) was calculated with the 7500 System SDS software version 1.3. Results were given as "relative expression" using glyceraldehyde-3-phosphate dehydrogenase (GAPDH, Hs99999905_m1) as the endogenous control and healthy control patient 005 [5] as the calibrator.

\section{Immunohistochemistry}

As described in our previous study, biopsied tissue was embedded in paraffin and sections were stained with either haematoxylin and eosin or Giemsa and van Gieson elastin [5]. For immunohistochemistry testing, biopsy sections were treated according to the standard protocol with the Leica BOND-MAX automated system using Leica Novocastra reagents (Biosystems, Nunningen, Switzerland). The standard protocol for immunohistochemistry was as follows: Dewaxing (AR9222), Epitope Retrieval Solution 2 (AR9640) and Bond Polymer Refine Detection Kit (DS9800) or Bond Polymer Refine Red Detection Kit (DS9390). The primary antibodies were 1F6 mouse monoclonal antibody to cluster of differentiation 4 (CD4, NCL-CD4-1F6, Novocastra) diluted 1:50; MJ1 mouse monoclonal antibody to CD20 (NCL-CD20MJ1, Novocastra) diluted 1:100; 11E3 mouse monoclonal antibody to CD79A (NCL-CD79a-225, Novocastra) diluted 1:125; AU-1 mouse monoclonal antibody to uroplakin 3 (UPK3, 345 M-14, Cell Marque, Rocklin, CA, USA) diluted 1:25; and PW31 mouse monoclonal antibody to KRT20 (NCL-L-CK20-561, Novocastra) diluted 1:200. Microphotographs were taken at $\times 20$ magnification with a UC30 colour camera mounted on a BX43 Olympus System Microscope. Images were processed by the CellF image analysis software (Olympus, Volketswil, Switzerland).

Blood handling and quantification of plasma $\operatorname{IgG}$ and IgA

Blood was collected in VACUETTE ${ }^{\circledR}$ Heparin tubes (Greiner Bio-One, St. Gallen, Switzerland) one day before biopsy. IgG and IgA concentrations were determined on a cobas $^{\circledR} 6000$ analyzer (Roche Diagnostics AG, Rotkreuz, Switzerland).
Urine handling and quantification of urinary immunoglobulins

Urine was obtained through urinary catheterization one day before the biopsy. Standard laboratory techniques included a routine urine status and a urine culture. Sterile urine was immediately centrifuged ( $5 \mathrm{~min}, 900 \mathrm{~g}$ ), and the supernatant was frozen at $-20{ }^{\circ} \mathrm{C}$ and subsequently stored at $-80{ }^{\circ} \mathrm{C}$. After thawing, protease inhibitor (Complete 04693116001, Roche Diagnostics AG, Rotkreuz, Switzerland) was added and $300 \mu \mathrm{l}$-aliquots were stored at $-80^{\circ} \mathrm{C}$. Concentration of urine creatinine was determined with the CREA plus kit from Roche/Hitachi on a Roche/Hitachi Modular System P Chemistry Analyzer (Roche Diagnostics AG, Rotkreuz, Switzerland) at Ilamed AG, Institute for Laboratory Medicine (Frauenfeld, Switzerland). For immunoglobulin quantification, urine aliquots were thawed and centrifuged for $15 \mathrm{~min}$ at $2,000 \mathrm{~g}$. Supernatants were adjusted to $\mathrm{pH} 7.4$ by adding a modified phosphate-buffered saline (PBS) buffer (final $1 \times$ concentration: $137 \mathrm{mM} \mathrm{NaCl}, 2.7 \mathrm{mM} \mathrm{KCl}$, $40 \mathrm{mM}$ phosphate buffer, $\mathrm{pH}$ 7.4). To quantify the protein concentration, three different dilutions were measured per urine sample. IgG and $\operatorname{IgA}$ concentrations were determined on a BD FACSCalibur flow cytometry instrument using Human Total IgG CBA Flex Set (No. 558679) and Human Total IgA CBA Flex Set (No. 558681) kits in accordance with the manufacturer's protocol (BD Biosciences, Allschwil, Switzerland).

\section{Statistics}

Statistical analyses were done in IBM $^{\circledR}$ SPSS ${ }^{\circledR}$ Statistics (version 19). A $p$ value of less than 0.05 was considered to indicate statistical significance. Tests of normality (ShapiroWilk) and homogeneity of variance (Levene statistic) were done with all continuous variables. The Kruskal-Wallis test was used for non-parametric data. Post hoc tests (MannWhitney U) identified differences between two groups, and the Bonferroni correction was applied. As such, a $p$ value of $0.016(0.05 / 3)$ indicated significance. For normally distributed data, analysis of variance (ANOVA) followed by Tukey's HSD post hoc tests were used to detect differences between the groups. Non-parametric receiver-operating characteristic (ROC) curves were generated for all biomarkers to plot the sensitivity against the false-positive rate (1-specificity). To accomplish this, biomarker values for BPS/IC ESSIC type 3C were compared to values of OAB combined with the healthy control group. The optimal cutoff for each biomarker was selected to maximize the sum of sensitivity and specificity [Youden index $=$ max. (selectivity + specificity-1] [12]. The accuracy of a biomarker to predict BPS/IC, defined as the average of sensitivity and specificity, was also calculated. 


\section{Results}

A total of 140 women were assessed for enrolment in the study; 106 were excluded from the analysis due to Hunner's negative forms of BPS/IC, an acute urinary tract infection or incomplete/insufficient tissue/urine samples or missing medical data. The 34 eligible women were grouped as follows: 15 in the BPS/IC ESSIC type 3C group, 11 in the $\mathrm{OAB}$ group and 8 in the control group. Demographic data of the study population are summarized in Table 1 .

High expression of B- and T-cell-specific genes and low expression of urothelium-specific genes in bladder biopsies of patients with BPS/IC ESSIC type 3C

For the BPS/IC ESSIC type 3C group, the statistical evaluation of the RT-qPCR data showed significantly high expression of the T- and B-cell-specific genes CTLA4, CD20, $C D 79 A$ and IGH@ and significantly low expression of the urothelium-specific genes UPK1B,UPK3A and KRT20 (Table 2) when compared with the other study groups. All seven gene expressions were relatively accurate biomarkers for BPS/IC ESSIC type 3C (Table 3).

\section{Abnormal urothelial gene expression in women with $\mathrm{OAB}$}

In the OAB group, the expression of the B- and T-cellspecific genes was low, which was similar to, but slightly above, the healthy control group (Table 2). The urothelial markers were in intermediate ranges between the other two groups, and the uroplakin gene $U P K 1 B$ reached significantly lower expression when compared to healthy controls (Table 2).
Formation of reactive lymphocyte aggregates in patients with BPS/IC ESSIC type 3C

To compare the gene expression data with characteristics at the cellular level, paraffin sections were analysed by immunohistochemistry. B-cells were stained by the CD79A marker. T-helper cells that promote the adaptive immune response and $\mathrm{B}$-cell activation were highlighted by the CD4 marker. Of 15 BPS/IC ESSIC type 3C patients, 13 had dense lymphocyte infiltrates in the bladder submucosa with preferential perivascular location. B-lymphocytes were found in the centre of the follicular aggregates and were surrounded by T-lymphocytes (Fig. 1). In contrast, the other two patient groups showed very limited lymphocyte infiltration with only occasional B- and T-cells in the submucosa. In the urothelium, CD4-positive cells were clearly elevated in the BPS/IC group as compared to the other groups, whereas CD79A-positive cells were scarce in all groups. Results similar to CD79A were observed for CD20 staining (data not shown).

\section{Abnormal urothelial staining in BPS/IC ESSIC type 3C patients}

Biopsy sections of healthy controls were characterized by a strong UPK3 staining with an enhancement in the umbrella cells facing the bladder lumen (Fig. 2). Approximately $60 \%$ of the umbrella cells also stained positive for KRT20 (Fig. 2). On the contrary, the urothelium of BPS/IC ESSIC type $3 \mathrm{C}$ patients was abnormal. In some cases it was thin, scarcely present or even absent, and there were instances in which it was lost during preparation because it did not adhere well to the submucosa (Fig. 2). KRT20 staining

Table 1 Demographic data

\begin{tabular}{|c|c|c|c|c|c|c|c|}
\hline Variable & $\begin{array}{l}\text { Descriptive } \\
\text { statistics }\end{array}$ & $\begin{array}{l}\text { BPS/IC ESSIC type } \\
3 \mathrm{C} \text { (group } 1, n=15)\end{array}$ & $\begin{array}{l}\text { OAB (group 2, } \\
n=11 \text { ) }\end{array}$ & $\begin{array}{l}\text { Healthy controls } \\
\text { (group } 3, n=8 \text { ) }\end{array}$ & $p$ value & Post hoc test & $p<0.05$ \\
\hline Subjects & White & 15 & 11 & 8 & NA & & \\
\hline Age & Mean & $66( \pm 11)$ & $51( \pm 20)$ & $47( \pm 10)$ & $p=0.006$ & $\begin{array}{l}\text { Gr. } 1 \& 2(p=0.035) \\
\text { Gr. } 1 \& 3(p=0.011) \\
\text { Gr. } 2 \& 3(p=0.773)\end{array}$ & $\begin{array}{l}\mathrm{S} \\
\mathrm{S} \\
\mathrm{NS}\end{array}$ \\
\hline BMI & Median & 26 (range $22-34$ ) & 29 (range 19-43) & 22 (range $20-27$ ) & $p=0.236$ & & \\
\hline Parity & Median & 3 (range $0-4$ ) & 2 (range $0-5)$ & 2 (range $0-3$ ) & $p=0.157$ & & \\
\hline Smokers & Smokers & $1(7 \%)$ & $2(18 \%)$ & $1(13 \%)$ & $p=0.673$ & & \\
\hline Urge incontinence & Yes & 5 & 7 & 1 & $p=0.067$ & & \\
\hline Urge incontinence & Several times a day & 0 & 5 & 0 & $p=0.002$ & $\begin{array}{l}\text { Gr. } 1 \& 2(p=0.007) \\
\text { Gr. } 1 \& 3(p=0.999) \\
\text { Gr. } 2 \& 3(p=0.045)\end{array}$ & $\begin{array}{l}\mathrm{S} \\
\mathrm{NS} \\
\mathrm{S}\end{array}$ \\
\hline Urge incontinence & Occasionally & 5 & 2 & 1 & $p=0.592$ & & \\
\hline
\end{tabular}

$G r$ group, $N A$ not applicable, $S$ significant, $N S$ not significant, $B M I$ body mass index 
Table 2 Statistics for gene expression (RT-qPCR)

\begin{tabular}{|c|c|c|c|c|c|c|c|}
\hline Variable & $\begin{array}{l}\text { Descriptive } \\
\text { statistics }\end{array}$ & $\begin{array}{l}\text { BPS/IC ESSIC type } 3 C^{\mathrm{a}} \\
\text { (group } 1, n=15 \text { ) }\end{array}$ & $\begin{array}{l}\mathrm{OAB}^{\mathrm{a}} \text { (group } 2, \\
n=11)\end{array}$ & $\begin{array}{l}\text { Healthy controls } \mathrm{s}^{\mathrm{a}} \\
\text { (group } 3, n=8 \text { ) }\end{array}$ & $p$ value & Post hoc test & $\begin{array}{l}\text { Bonferroni } \\
\text { correction } \\
(p<0.016)\end{array}$ \\
\hline \multirow[t]{5}{*}{ CD79A_qPCR } & Mean & 11.16 & 0.36 & 0.29 & \multirow[t]{5}{*}{$p<0.0001$} & Gr. $1 \& 2(p<0.0001)$ & $\mathrm{S}$ \\
\hline & $\sigma^{\mathrm{b}}$ & 9.17 & 0.56 & 0.47 & & Gr. $1 \& 3(p<0.0001)$ & $\mathrm{S}$ \\
\hline & Median & 8.94 & 0.12 & 0.06 & & Gr. $2 \& 3(p=0.215)$ & NS \\
\hline & Minimum & 1.30 & 0.02 & 0.01 & & & \\
\hline & Maximum & 40.90 & 1.96 & 1.22 & & & \\
\hline \multirow[t]{5}{*}{ CD20_qPCR } & Mean & 14.11 & 0.71 & 0.21 & \multirow[t]{5}{*}{$p<0.0001$} & Gr. $1 \& 2(p<0.0001)$ & $\mathrm{S}$ \\
\hline & $\sigma^{\mathrm{b}}$ & 9.71 & 1.72 & 0.46 & & Gr. $1 \& 3(p<0.0001)$ & $\mathrm{S}$ \\
\hline & Median & 13.58 & 0.16 & 0.01 & & Gr. $2 \& 3(p=0.240)$ & NS \\
\hline & Minimum & 1.50 & 0.00 & 0.00 & & & \\
\hline & Maximum & 33.78 & 5.85 & 1.31 & & & \\
\hline \multirow[t]{5}{*}{ CTLA4_qPCR } & Mean & 13.62 & 0.78 & 0.53 & \multirow[t]{5}{*}{$p<0.0001$} & Gr. $1 \& 2(p<0.0001)$ & $\mathrm{S}$ \\
\hline & $\sigma^{\mathrm{b}}$ & 10.77 & 0.55 & 0.51 & & Gr. $1 \& 3(p<0.0001)$ & $\mathrm{S}$ \\
\hline & Median & 10.13 & 0.57 & 0.25 & & Gr. $2 \& 3(p=0.107)$ & NS \\
\hline & Minimum & 2.39 & 0.28 & 0.14 & & & \\
\hline & Maximum & 35.67 & 1.95 & 1.55 & & & \\
\hline \multirow[t]{5}{*}{ IGH@_qPCR } & Mean & 44.11 & 0.75 & 1.01 & \multirow[t]{5}{*}{$p<0.0001$} & Gr. $1 \& 2(p<0.0001)$ & $\mathrm{S}$ \\
\hline & $\sigma^{\mathrm{b}}$ & 53.58 & 0.91 & 1.83 & & Gr. $1 \& 3(p<0.0001)$ & $\mathrm{S}$ \\
\hline & Median & 23.71 & 0.33 & 0.22 & & Gr. $2 \& 3(p=0.563)$ & NS \\
\hline & Minimum & 1.30 & 0.01 & 0.00 & & & \\
\hline & Maximum & 158.19 & 2.79 & 5.28 & & & \\
\hline \multirow[t]{5}{*}{ KRT20_qPCR } & Mean & 0.22 & 1.84 & 2.31 & \multirow[t]{5}{*}{$p<0.0001$} & Gr. $1 \& 2(p<0.0001)$ & $\mathrm{S}$ \\
\hline & $\sigma^{\mathrm{b}}$ & 0.63 & 1.97 & 1.69 & & Gr. $1 \& 3(p<0.0001)$ & $\mathrm{S}$ \\
\hline & Median & 0.00 & 1.02 & 2.13 & & Gr. $2 \& 3(p=0.283)$ & NS \\
\hline & Minimum & 0.00 & 0.22 & 0.27 & & & \\
\hline & Maximum & 2.32 & 6.69 & 5.78 & & & \\
\hline \multirow[t]{5}{*}{$U P K 1 B \_\mathrm{qPCR}$} & Mean & 0.13 & 0.87 & 1.37 & \multirow[t]{5}{*}{$p<0.0001$} & Gr. $1 \& 2(p<0.0001)$ & $\mathrm{S}$ \\
\hline & $\sigma^{\mathrm{b}}$ & 0.18 & 0.41 & 0.32 & & Gr. $1 \& 3(p<0.0001)$ & $\mathrm{S}$ \\
\hline & Median & 0.03 & 0.69 & 1.44 & & Gr. $2 \& 3(p=0.013)$ & $\mathrm{S}$ \\
\hline & Minimum & 0.00 & 0.27 & 0.80 & & & \\
\hline & Maximum & 0.65 & 1.52 & 1.78 & & & \\
\hline \multirow[t]{5}{*}{$U P K 3 A$ _qPCR } & Mean & 0.13 & 1.02 & 1.97 & \multirow[t]{5}{*}{$p<0.0001$} & Gr. $1 \& 2(p<0.0001)$ & $\mathrm{S}$ \\
\hline & $\sigma^{\mathrm{b}}$ & 0.30 & 0.64 & 0.81 & & Gr. $1 \& 3(p<0.0001)$ & $\mathrm{S}$ \\
\hline & Median & 0.03 & 1.02 & 2.13 & & Gr. $2 \& 3(p=0.021)$ & NS \\
\hline & Minimum & 0.00 & 0.28 & 0.73 & & & \\
\hline & Maximum & 1.13 & 2.50 & 3.27 & & & \\
\hline
\end{tabular}

$G r$ group, $S$ significant, $N S$ not significant

${ }^{a}$ Relative gene expression

${ }^{\mathrm{b}} \sigma$ : standard deviation

was absent in all of the 13 investigated BPS/IC ESSIC type $3 \mathrm{C}$ patients, and UPK 3 staining was absent or weak without the characteristic umbrella cell staining (in 13 of 14 investigated patients). In the $\mathrm{OAB}$ group, the urothelium was clearly present and KRT20 staining was comparable to the healthy controls; however, in six of ten patients the UPK3 staining was less intense (Fig. 2).
High levels of urinary immunoglobulins in women with BPS/IC ESSIC type 3C

The obvious accumulation of CD79A- and CD20-positive Bcells indicated a local immune response in the BPS/IC ESSIC type $3 \mathrm{C}$ group. The quantification of secretory $\operatorname{IgA}$ and $\operatorname{IgG}$ showed a significantly high level of these immunoglobulins in 
Table 3 Cut-off values and accuracy of biomarkers for BPS/IC ESSIC type 3C

\begin{tabular}{lllllllll}
\hline Biomarker & $\begin{array}{l}\text { Cut-off } \\
\text { value }^{\mathrm{c}}\end{array}$ & $\begin{array}{l}\text { AUROC } \\
(95 \% \mathrm{CI})\end{array}$ & $\begin{array}{l}\text { Youden } \\
\text { index }\end{array}$ & Accuracy & Sensitivity & Specificity & $\begin{array}{l}\text { Positive predictive } \\
\text { value }\end{array}$ & $\begin{array}{l}\text { Negative predictive } \\
\text { value }\end{array}$ \\
\hline CD79A & 1.26 & $0.99(0.99-1.00)$ & 0.95 & 0.97 & 1 & 0.95 & 0.94 & 1 \\
CD20 & 1.41 & $0.98(0.95-1.00)$ & 0.95 & 0.97 & 1 & 0.95 & 0.94 & 1 \\
CTLA4 & 2.17 & $1.00(1.00-1.00)$ & 1 & 1 & 1 & 1 & 1 & 1 \\
IGH@ & 2.23 & $0.97(0.93-1.00)$ & 0.83 & 0.91 & 0.93 & 0.89 & 0.88 & 0.94 \\
KRT20 & 0.13 & $0.94(0.84-1.00)$ & 0.87 & 0.93 & 0.87 & 1 & 1 & 0.95 \\
UPK1B & 0.43 & $0.98(0.94-1.00)$ & 0.88 & 0.94 & 0.93 & 0.95 & 0.93 & 0.95 \\
UPK3A & 0.44 & $0.95(0.88-1.00)$ & 0.83 & 0.91 & 0.93 & 0.89 & 0.88 & 0.94 \\
IgA & 194.36 & $0.97(0.91-1.00)$ & 0.83 & 0.91 & 0.93 & 0.89 & 0.88 & 0.94 \\
IgG & 246.17 & $0.96(0.89-1.00)$ & 0.89 & 0.95 & 1 & 0.89 & 0.88 & 1 \\
\hline
\end{tabular}

$A U R O C$ area under ROC curve, $C I$ confidence interval

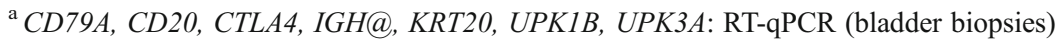

${ }^{\mathrm{b}} \mathrm{IgA}$ and IgG: enzyme-linked immunosorbent assay quantification (urine)

${ }^{\mathrm{c}}$ Cut-off values: RT-qPCR: relative gene expressions; IgA and $\mathrm{IgG}: \mathrm{ng} / \mu \mathrm{mol}$ creatinine

the urine (Table 4). This was not the case for the other two study groups, although values for $\mathrm{OAB}$ were above healthy control levels. Blood IgA and IgG levels were not significantly different for the three study groups (data not shown). Both urinary $\operatorname{IgA}$ and $\operatorname{IgG}$ were relatively accurate biomarkers for BPS/IC ESSIC type 3C (Table 3).

Elevated number of mast cells in detrusor muscle

All patients with BPS/IC ESSIC type 3C had elevated mast cell counts in the detrusor which was associated with high
B- and T-cell marker expression. However, this association was not found in the other groups. While the B- and T-cell marker expression was low for both groups, 3 of $11 \mathrm{OAB}$ patients and 4 of 8 healthy controls had elevated mast cell counts in the detrusor.

\section{Discussion}

Gene expression analysis of four T- and B-cell markers (CTLA4,CD20,CD79A and IGH@) and three urothelial

\section{Ulcerative IC}
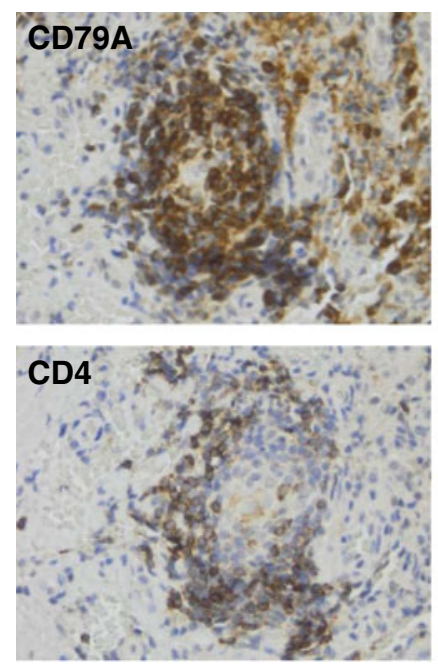

\section{OAB}
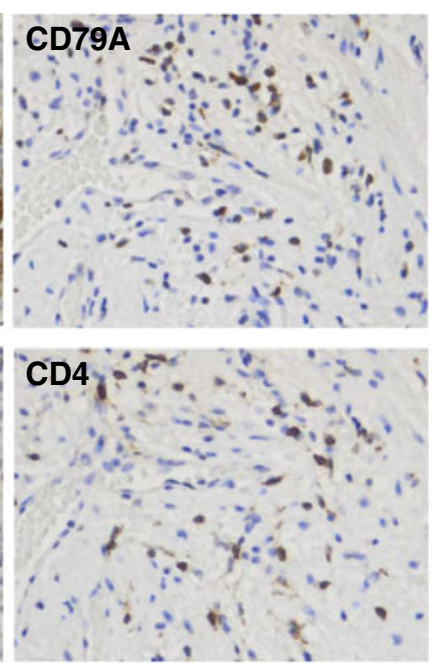

\section{Healthy Control}
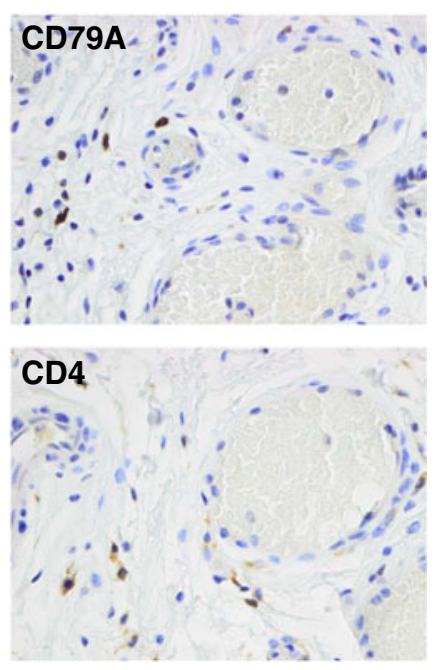

Fig. 1 B- and T-lymphocyte staining in bladder submucosa. Immunohistochemistry with paraffin sections of bladder biopsies using primary monoclonal antibodies against CD4 (T-cell marker) and CD79A (Bcell marker). Representative pictures are shown for the three investigated groups, BPS/IC ESSIC type 3C (group 1), OAB (group 2) and healthy controls (group 3). The respective CD79A and CD4 stainings are from the same biopsy location. All pictures are at the same magnification. The lymphocyte aggregate shown has a size of $100 \mu \mathrm{m} \times$ $170 \mu \mathrm{m}$ and lymphocyte counts of approximately 200 cells; this results in a lymphocyte density of $1.2 \times 10^{4}$ cells $/ \mathrm{mm}^{2}$ 
Ulcerative IC
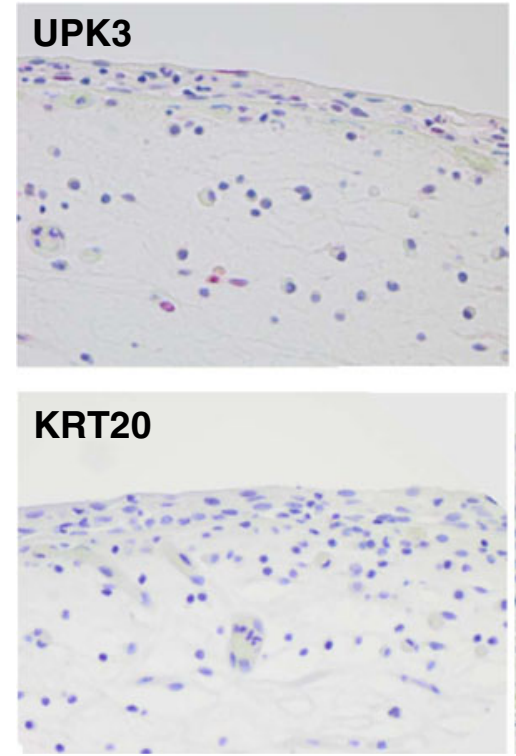

Fig. 2 Staining of urothelium-specific proteins in bladder biopsies. Immunohistochemistry with paraffin sections of bladder biopsies using primary monoclonal antibodies against two urothelial markers, UPK3 (uroplakin 3) and KRT20 (cytokeratin 20). Representative pictures are

markers (UPK1B, UPK3A and KRT20) revealed significant differences between the BPS/IC ESSIC type $3 \mathrm{C}$ group and the two control groups. High concentrations of $\operatorname{IgG}$ and $\operatorname{Ig} \mathrm{A}$ in urine, but not in blood, were associated with high expression of lymphocyte-specific genes and follicular B- and Tcell aggregates in the submucosa and were consistent with our hypothesis that there is a local immune response with Bcell activation in cases of BPS/IC ESSIC type 3C. In addition, urothelium-specific gene expressions were low in BPS/IC ESSIC type 3C. One urothelial marker $(U P K 1 B)$ even reached
OAB

\section{Healthy Control}
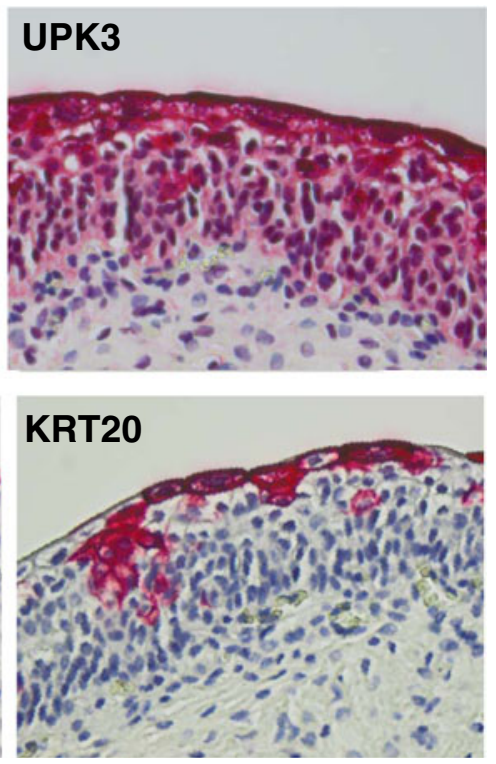

shown for the three investigated groups, BPS/IC ESSIC type 3C (group 1), OAB (group 2) and healthy controls (group 3). The respective UPK3 and KRT20 stainings are from the same biopsy location. All pictures are at the same magnification

significantly lower expression for non-inflammatory $\mathrm{OAB}$ when compared to the healthy control group. For most other gene expression markers and the urine immunoglobulin concentrations, the $\mathrm{OAB}$ values lie between those of the healthy control group and the severe BPS/IC form.

The results for BPS/IC ESSIC type 3C are consistent with earlier findings that describe severe inflammatory infiltrates and an abnormal urothelium for this disease subtype $[1,2,6-8,13-16]$. In contrast to previous studies, though, we provided a subset of quantifiable in vivo gene expression

Table 4 Statistics for immunoglobulin concentration in urine

\begin{tabular}{|c|c|c|c|c|c|c|c|}
\hline Variable & $\begin{array}{l}\text { Descriptive } \\
\text { statistics }\end{array}$ & $\begin{array}{l}\text { BPS/IC ESSIC type } 3 \mathrm{C}^{\mathrm{a}} \\
\text { (group } 1, n=15 \text { ) }\end{array}$ & $\begin{array}{l}\mathrm{OAB}^{\mathrm{a}}(\text { group } 2, \\
n=11)\end{array}$ & $\begin{array}{l}\text { Healthy controls } \\
\text { (group } 3, n=8 \text { ) }\end{array}$ & $p$ value & Post hoc test & $\begin{array}{l}\text { Bonferroni correction } \\
(p<0.016)\end{array}$ \\
\hline \multirow[t]{5}{*}{$\operatorname{Ig} \mathrm{A}$} & Mean & 693 & 150 & 92 & \multirow[t]{5}{*}{$p<0.0001$} & Gr. $1 \& 2(p<0.0001)$ & $\mathrm{S}$ \\
\hline & $\sigma^{\mathrm{b}}$ & 530 & 57 & 56 & & Gr. $1 \& 3(p<0.0001)$ & $\mathrm{S}$ \\
\hline & Median & 518 & 141 & 78 & & Gr. $2 \& 3(p=0.058)$ & NS \\
\hline & Minimum & 136 & 69 & 27 & & & \\
\hline & Maximum & 1,781 & 251 & 192 & & & \\
\hline \multirow[t]{5}{*}{$\operatorname{IgG}$} & Mean & 1,317 & 213 & 115 & \multirow[t]{5}{*}{$p<0.0001$} & Gr. $1 \& 2(p<0.0001)$ & $\mathrm{S}$ \\
\hline & $\sigma^{\mathrm{b}}$ & 1,466 & 285 & 75 & & Gr. $1 \& 3(p<0.0001)$ & $\mathrm{S}$ \\
\hline & Median & 672 & 98 & 115 & & Gr. $2 \& 3(p=0.741)$ & NS \\
\hline & Minimum & 253 & 33 & 17 & & & \\
\hline & Maximum & 5,909 & 999 & 239 & & & \\
\hline
\end{tabular}

Gr. group, $S$ significant, $N S$ not significant

${ }^{a}$ ng immunoglobulin $/ \mu$ mol creatinine

${ }^{\mathrm{b}} \sigma$ : standard deviation 
markers for both the inflammatory and the urothelial aspects of the disease. Measurement of these markers may aid in the detection of BPS/IC ESSIC type 3C.

Although urinary immunoglobulins have been detected in earlier studies [17], this is the first report on BPS/IC ESSIC type $3 \mathrm{C}$ to show significantly high levels of antibodies in urine.

$\mathrm{OAB}$ and $\mathrm{BPS} / \mathrm{IC}$ are regarded as different syndromesurgency is considered the hallmark symptom of $\mathrm{OAB}$ and pain is the hallmark symptom of BPS/IC [18]. In our study, $\mathrm{OAB}$ patients with non-inflammatory cystoscopic findings served as a control group. Our intention was to determine if the marker values for the $\mathrm{OAB}$ group were more similar to those of the BPS/IC group, the healthy group, or if the values were at intermediate ranges between these two groups since other recently published studies found an overlap between $\mathrm{OAB}$ and BPS/IC on both a clinical and diagnostic level [9-11, 18]. Under this assumption, the results we obtained for the $\mathrm{OAB}$ group can be explained.

The low expression of the urothelial genes in BPS/IC type 3C might be due to a loss of urothelium within the Hunner's lesion, which has also been described by other researchers [19, 20]. However, in our previous study [5], two biopsies of a Hunner's positive BPS/IC bladder were analysed - one from the transition zone Hunner's lesion/non-lesion and the second from a normal-looking area of the bladder. The urothelial genes UPK1B (210064_s_at) and KRT20 (213953_at) were shown to be expressed at significantly lower levels, even in the non-affected, normal-looking bladder tissue [5].

Only a few studies addressed the impact of urothelium integrity on $\mathrm{OAB}$; however, Liu et al. [10] recently found that the urothelial barrier function was normal in $\mathrm{OAB}$ patients. Conversely, findings from our study showed significantly low expression of one urothelial marker (UPK1B) in non-inflammatory OAB patients compared to the healthy control group. Limited comparisons can be made between these two studies since Liu et al. used patients with stress urinary incontinence as controls rather than patients free of bladder symptoms. Moreover, they performed integrated image sections measuring immunofluorescence rather than comprehensive quantitative gene expressions, and they looked at different urothelial markers (zonula occludens-1 and E-cadherin rather than UPK1B, UPK3A and KRT20).

Even though efforts have been made [13, 21], there are currently no commonly accepted markers to diagnose BPS/IC. Furthermore, there are only invasive techniques to differentiate Hunner's positive from Hunner's negative $\mathrm{BPS} / \mathrm{IC}$. It is likely that the $\operatorname{IgA}$ and $\operatorname{IgG}$ urine findings are due to the presence of lymphocyte infiltrates in the submucosa and would not be elevated if the histology were normal. As part of our future research, we plan to investigate whether elevated urinary IgA and IgG levels are indicative of Hunner's lesion, which would mean a non-invasive technique to identify this disease subtype would be available. Since an association between BPS/IC and autoimmune diseases has been suggested [22], determining the specificity of the urinary immunoglobulins would likely give new information on the catalyst and the pathophysiology of this local immune reaction.

The B-cell markers $C D 79 A$ and $C D 20$ are similarly expressed (Table 2); therefore, quantification of one of these representative markers should be sufficient. The elevated number of B-cells in the bladder wall is an important consideration for prospective therapeutic approaches to BPS/IC ESSIC type 3C. Rituximab, a monoclonal antibody specific for CD20, selectively destroys B-cells [23]. Given our findings of high $C D 20$ expression in Hunner's positive BPS/IC, we would propose treatment with rituximab, a treatment which currently is approved for use with non-Hodgkin's lymphoma, rheumatoid arthritis and ANCA (anti-neutrophil cytoplasmic antibodies)-associated vasculitis [23, 24]. By tracking changes in gene expression, we may be able to quantify and monitor therapy progress and outcomes.

With regard to study limitations, the low number of study participants was largely a consequence of a small pool of eligible patients at one recruitment centre and a relatively rare condition. Furthermore, the healthy controls were patients hospitalized for gynaecological surgery and underwent cystoscopy and bladder biopsy solely for study purposes. For this reason, patient groups could not be matched by age, which could signify a potential source of bias. Similar to other studies [25, 26], patients with Hunner's lesions were older than the comparison groups.

We chose to exclude patients with Hunner's negative BPS/IC since they make up a large and heterogeneous group. This study was intended as an initial investigative step to find biomarkers; therefore, we focused our research efforts on the small homogeneous group of patients with Hunner's positive BPS/IC who could easily be identified with cystoscopy. For future studies, a careful subdivision of the heterogeneous Hunner's negative BPS/IC group based on the ESSIC grading system (i.e. cystoscopy and biopsy data) would be required.

Tests of sensitivity and specificity showed that all markers were accurate biomarkers for BPS/IC ESSIC type 3C (Table 3). However, these results were obtained from small, homogeneous subgroups of patients. Further analyses are planned with a larger cohort of patients, including those with Hunner's negative BPS/IC.

In summary, this study identified seven quantifiable gene expression markers which can describe the current stage of the clinically defined subtype of BPS/IC ESSIC type 3C. Objective, measurable results can be generated within 2 days and more focused monitoring of therapy outcomes could be carried out. Further research is needed to develop more effective strategies for treating Hunner's positive BPS/IC, 
such as targeted therapy with monoclonal antibodies against B-cells. Detection of elevated urinary $\operatorname{IgA}$ and $\operatorname{IgG}$ levels could be a non-invasive technique to diagnose the subtype BPS/IC ESSIC type 3C representing $~ 10 \%$ of all BPS/IC patients [20]. Lastly, since the results for the non-inflammatory $\mathrm{OAB}$ group were in intermediate ranges between BPS/IC ESSIC type 3C and the healthy control groups, future research efforts ought to assess if BPS/IC and OAB are related.

Acknowledgements The Astellas European Foundation supported this work with the Urology Prize Fund 2007. Further funding was from grants of Kantonsspital Frauenfeld, Spital Thurgau AG, Switzerland. The funds were freely available for basic research and no claims on study design, data collection, analysis or interpretation or writing of the report were raised. We thank Nicola Späth for expert technical assistance and Ronald Bräutigam, Susanne Huber-Reuter and Thomas Luginbühl for performing surgical biopsies. Christine Kressibucher and the team of the Institute for Pathology, Spital Thurgau AG (Münsterlingen, Switzerland) are acknowledged for providing technical support. We acknowledge Susanne Forst, Nicole Graf and Susanne Härri for their contributions.

\section{Conflicts of interest None.}

Open Access This article is distributed under the terms of the Creative Commons Attribution License which permits any use, distribution, and reproduction in any medium, provided the original author(s) and the source are credited.

\section{References}

1. Harrington DS, Fall M, Johansson SL (1990) Interstitial cystitis: bladder mucosa lymphocyte immunophenotyping and peripheral blood flow cytometry analysis. J Urol 144:868-871

2. Johansson SL, Fall M (1990) Clinical features and spectrum of light microscopic changes in interstitial cystitis. J Urol 143:1118-1124

3. Hanno PM, Landis JR, Matthews-Cook Y et al (1999) The diagnosis of interstitial cystitis revisited: lessons learned from the National Institutes of Health Interstitial Cystitis Database study. J Urol 161:553-557

4. van de Merwe JP, Nordling J, Bouchelouche P et al (2008) Diagnostic criteria, classification, and nomenclature for painful bladder syndrome/interstitial cystitis: an ESSIC proposal. Eur Urol 53:60-67

5. Gamper M, Viereck V, Geissbühler V et al (2009) Gene expression profile of bladder tissue of patients with ulcerative interstitial cystitis. BMC Genomics 10:199

6. Slobodov G, Feloney M, Gran C et al (2004) Abnormal expression of molecular markers for bladder impermeability and differentiation in the urothelium of patients with interstitial cystitis. J Urol 171:1554-1558

7. Zeng Y, Wu XX, Homma Y et al (2007) Uroplakin III-delta4 messenger RNA as a promising marker to identify nonulcerative interstitial cystitis. J Urol 178:1322-1327, discussion 1327
8. Ogawa T, Homma T, Igawa Y et al (2010) CXCR3 binding chemokine and TNFSF14 over expression in bladder urothelium of patients with ulcerative interstitial cystitis. J Urol 183:12061212

9. Cartwright R, Afshan I, Derpapas A et al (2011) Novel biomarkers for overactive bladder. Nat Rev Urol 8:139-145

10. Liu HT, Shie JH, Chen SH et al (2012) Differences in mast cell infiltration, E-cadherin, and zonula occludens-1 expression between patients with overactive bladder and interstitial cystitis/bladder pain syndrome. Urology 80(1):225.e13-225.e18

11. Chung SD, Liu HT, Lin H et al (2011) Elevation of serum creactive protein in patients with $\mathrm{OAB}$ and IC/BPS implies chronic inflammation in the urinary bladder. Neurourol Urodyn 30:417420

12. Fluss R, Faraggi D, Reiser B (2005) Estimation of the Youden Index and its associated cutoff point. Biom J 47:458-472

13. Tyagi P, Killinger K, Tyagi V et al (2012) Urinary chemokines as noninvasive predictors of ulcerative interstitial cystitis. J Urol 187:2243-2248

14. Blalock EM, Korrect GS, Stromberg AJ et al (2012) Gene expression analysis of urine sediment: evaluation for potential noninvasive markers of interstitial cystitis/bladder pain syndrome. J Urol 187:725-732

15. Yang W, Kim Y, Kim TK et al (2012) Integration analysis of quantitative proteomics and transcriptomics data identifies potential targets of frizzled- 8 protein-related antiproliferative factor in vivo. BJU Int 110:E1138-E1146

16. Logadottir Y, Hallsberg L, Fall M et al (2013) Bladder pain syndrome/interstitial cystitis ESSIC type 3C: high expression of inducible nitric oxide synthase in inflammatory cells. Scand J Urol 47:52-56

17. Keay S, Zhang CO, Trifillis AL et al (1997) Urine autoantibodies in interstitial cystitis. J Urol 157:1083-1087

18. Elliott CS, Payne CK (2012) Interstitial cystitis and the overlap with overactive bladder. Curr Urol Rep 13:319-326

19. Peeker R, Aldenborg F, Fall M (2000) Complete transurethral resection of ulcers in classic interstitial cystitis. Int Urogynecol J Pelvic Floor Dysfunct 11:290-295

20. Payne RA, O'Connor RC, Kressin M et al (2009) Endoscopic ablation of Hunner's lesions in interstitial cystitis patients. Can Urol Assoc J 3:473-477

21. Corcoran AT, Yoshimura N, Tyagi V et al (2013) Mapping the cytokine profile of painful bladder syndrome/interstitial cystitis in human bladder and urine specimens. World J Urol 31:241-246

22. van de Merwe JP (2007) Interstitial cystitis and systemic autoimmune diseases. Nat Clin Pract Urol 4:484-491

23. Boross P, Leusen JH (2012) Mechanisms of action of CD20 antibodies. Am J Cancer Res 2:676-690

24. Edwards JC, Cambridge G (2006) B-cell targeting in rheumatoid arthritis and other autoimmune diseases. Nat Rev Immunol 6:394 403

25. Peeker R, Fall M (2002) Toward a precise definition of interstitial cystitis: further evidence of differences in classic and nonulcer disease. J Urol 167:2470-2472

26. Erickson DR, Tomaszewski JE, Kunselman AR et al (2008) Urine markers do not predict biopsy findings or presence of bladder ulcers in interstitial cystitis/painful bladder syndrome. J Urol 179:1850-1856 